\title{
Un acercamiento a la historia de la Cirugía de la Mano
}

\author{
J. Cantero Martínez \\ Antiguo Médico-Jefe de la Permanence Chirurgicale de Longeraie de Lausanne (Suiza) \\ MIEMBRO DE HONOR DE LA SECMA \\ Correspondencia: \\ Dr. José Cantero Martínez \\ e-mail: 972622496@telefonica.net
}

Palabras clave: Historia de la Cirugía de la Key words: History of Hand Surgery. Mano.

Rev. Iberam. Cir. Mano - Vol. 37 • Núm. 1 • Mayo 2009 (8-16)

Y digo un acercamiento, porque sería pretencioso por mi parte el intentar abarcar toda la historia de la Cirugía de la Mano en el espacio de un artículo. Así pues, me limitaré hacer un amplio resumen de lo que ha sido, a través de los tiempos, esta materia.

Podríamos preguntarnos desde cuándo la Cirugía de la Mano existe como entidad independiente de las otras ramas de la cirugía. La mayoría de los nombres a ella asociados, corresponden en realidad a personajes cuya principal actividad quirúrgica era otra que la que nos ocupa. Por ello es interesante examinar, de qué forma la patología de la mano ha interesado a nuestros antepasados a través del tiempo.

Desde que el hombre practica la cirugía, el tratamiento de la mano herida ha estado siempre entre sus preocupaciones, dado que la mano siempre ha estado expuesta a numerosos traumatismos. Pero se reducía, generalmente, a simples gestos, con frecuencia, rápidos y dolorosos. Hasta hace muy pocos años - mediados del siglo pasado - , la Cirugía de la Mano era considerada por muchos cirujanos como «pequeña cirugía», dejado su tratamiento en las manos inexpertas del último recién llegado al Servicio de Cirugía.

La mano tiene, por el contrario, unas características anatómicas específicas en el hombre.
Además de ser un elemento primordial de la prensión, gracias a la oposición del pulgar, tiene en el sentido del tacto una tal precisión que sirve de lectura al ciego, es capaz de hacer música y sirve de lenguaje al sordomudo. Por ello Aristóteles (354-322 a.C.) la calificó de «instrumento de los instrumentos» y Galeno (129200) decía que «el hombre piensa y gobierna a las demás criaturas precisamente porque tiene manos».

Cuando Amenhotep IV, más conocido como Ajenaton o Akenaton, décimo faraón de la XVIII dinastía de Egipto, que reinó entre el 1353 y 1336 a.C., establece una nueva religión, hace grabar en las tumbas y templos del Alto Egipto un cierto número de emblemas religiosos, entre los cuales se encuentra la adoración del sol. Los rayos de estos soles se terminan en unas manos estilizadas, protectoras de las criaturas. En el fondo de la tumba de Ramsés VI, quinto faraón de la XX dinastía de Egipto, que reinó entre el 1145 y 1137 a.C., se encuentra un símbolo humano con los dos brazos levantados por encima de la cabeza.

A juzgar por las marcas de manos encontradas entre las pinturas rupestres de algunas grutas, tales como las de Lescaut y de Pech-Merle en Francia, o las encontradas en Borneo, formando 
aquí un grupo de manos en negativo, o las de la Cueva de las Manos en Patagonia (Argentina), realizadas entre 11000 y 7500 a.C., la mano tenía en la Prehistoria una cierta significación religiosa o esotérica. Algunas muestran dedos amputados, que podrían tener un significado de duelo.

Según los últimos estudios practicados por Mario Capecchi, nacido en Verona en 1937 y premio Nóbel de Medicina en 2007, se presume que el hombre, tal como es actualmente existiría ya hace dos o tres millones de años, y su mano ha evolucionado con él. El descubrimiento del fuego provocaría sin duda quemaduras $\mathrm{y}$, por lo tanto infecciones, algunas de las cuales acabarían en amputaciones.

Quizás sea en la antigua Grecia donde podemos encontrar las primeras manifestaciones de una verdadera Cirugía de la Mano. Hipócrates (460-377 a.C.) sabía ya cómo reducir luxaciones de los dedos, y cómo reducir e inmovilizar fracturas de los mismos. Caius Plinius Secundus, llamado «Plinio el Viejo» (27-29), habla ya de un amputado aparejado con una mano artificial. Claudio Galeno (129-200), que fue sin duda, con Hipócrates, la máxima autoridad en Medicina en la Grecia antigua, describe ya las infecciones de la mano y establece la diferencia entre arterias y venas. Pablo de Egina (628-690), de la Escuela de Alejandría, fue seguramente el último de una gran sucesión de médicos que han marcado los principales periodos de la medicina griega clásica. Su libro, en siete volúmenes, «Epitomae Medicae» fue acogido con gran entusiasmo y traducido rápidamente al árabe. En su sexto volumen describe las afecciones quirúrgicas de partes blandas y del hueso, así como las quemaduras en las manos.

Comienza entonces, la gran época árabe con Abu Bakú Muhammad ibn Zakariya al-Razi, conocido como Rhazés (865-925), médico persa, fundador de un gran hospital en Bagdad, donde acuden estudiantes de todo el mundo árabe. Dio mucha importancia al examen clínico para llegar a un diagnóstico y al estudio psicológico del enfermo. Escribió 184 libros científicos, de los cuales 61 dedicados a la Medicina. Otro gran nombre de la medicina árabe es sin lugar a dudas Abú Ali al-Husayn ibn Abd Allah ibn Sina, más conocido por su nombre latinizado de Avicena (980-1037), considerado como el más gran- de de los médicos de todos los tiempos. Escribió más de 250 libros, entre ellos «El Canon de la Medicina» y «El Libro de las Curaciones».

En «Al-Andalus» florece una época fantástica de desarrollo de la Medicina. Hay que destacar en primer lugar a Abú al Qasim al-Zahrawi, conocido bajo el nombre de Abulcasis, nacido en Zahra (Córdoba) en 936, y allí murió en 1013. Escribió un importante tratado de medicina: el «Al-Tasrif». En su tercera parte trata de cauterizaciones, de fracturas, describe la hemiplejía de origen traumático. Imaginó una férula de contención de las fracturas de los metacarpianos, que fue utilizada hasta el siglo XIX. Enseñó cómo suturar las heridas desde dentro, con el fin de dejar cicatrices menos visibles. Practicó amputaciones y utilizó el método de reducción de luxaciones de hombro, semejante al conocido de Kocher.

Otros nombres de la medicina hispano-árabe son Avenzoar (1091-1163) y Averroes (11261198), aunque, sin duda, menos interesados por los problemas de las manos.

Pero es en el siglo XVI, con el flamenco Andrea Vesalio (1514-1564) cuando se inicia el periodo de los grandes descubrimientos anatómicos, gracias a la disección de cadáveres humanos y no de animales. «De Humanis Corporis Fabrica», corrigió errores arrastrados desde Galeno, durante trece siglos.

Los alumnos de Vesalio continuaron su obra. Eran tanto cirujanos como anatomistas, tales como Gabriele Falloppio (1523-1562), o William Harvey (1578-1657), descubridor de la circulación sanguínea en el hombre. Estudió en Padova, donde enseñó Falloppio y donde fue alumno de Girolamo Fabrice. Estos descubridores participan de forma activa en las adquisiciones del Renacimiento en muchos dominios, mientras que, al mismo tiempo, en Francia, la cirugía queda fuera de la enseñanza académica.

En Suiza, Theophrastus Bombastus ab Hohenheim, llamado Paracelsius (14931541), profesor de la universidad de Basilea, alquimista y médico, dice que «los humores no intervienen en la supuración de las heridas: mantenedlas limpias y preservarlas de los enemigos exteriores».

A Ambroise Paré (1509-1590), médico francés, se le considera como el padre de la cirugía 
moderna. De condición humilde, no tuvo acceso a una formación académica. Ignoraba el griego y el latín, por lo que sus obras las escribía en francés. Empezó su carrera como cirujano-barbero. A los 17 años consiguió ser admitido en el Hôtel-Dieu de Paris. Participa como cirujano militar en las campañas de Italia. Aplicó la ligadura de vasos sanguíneos en las amputaciones. Describió la utilización de prótesis en miembros amputados, así como el uso de férulas para enderezar los dedos, después de la resección de cicatrices retráctiles.

Felix Wurtz (1518-1575), cirujano pediatra de Basilea, describe en su libro «Practica der Wundtartzney» cómo curar las heridas de todo el cuerpo y pone de manifiesto los ciegos dogmatismos del pasado.

El siglo XVII nos trae contribuciones muy interesantes. No hay más que recordar como prueba evidente de ello «La Lección de Anatomía» de Rembrand (1609-1669). El suizo, también de Basilea, Felix Plater (1536-1614) describe en 1614, dos siglos antes que Dupuytren, una retracción de la aponeurosis palmar. Ya hemos mencionado más arriba, entre los alumnos de Vesalio, a William Harvey y su importante descubrimiento y descripción de la circulación sanguínea en el hombre. Sin embargo, pocas cosas pueden atribuirse específicamente a la mano durante este siglo XVII. La mayoría de los cirujanos con algún renombre, habían participado en innumerables batallas, adquiriendo en ellas una experiencia lejos de la tutela de la Facultad. Se trataba sobre todo de amputaciones, infecciones, inmovilización de fracturas, etc.

En Francia, Pierre Dionis (1673-1718), habla de desviaciones de los dedos y de tenosinovitis purulentas. La mayoría de sus trabajos fueron quemados durante la Revolución Francesa, probablemente porque fue llamado en su tiempo por Luis XIV para enseñar «La Anatomía según la circulación de la sangre», mientras que la $\mathrm{Fa}-$ cultad de Medicina se oponía al descubrimiento de dicha circulación hecho por Harvey.

La fundación de la Academia Real de Cirugía por Luis XV , en 1731, marca sin duda el comienzo de la moderna cirugía francesa. Tanto Georges Maréchal (1658-1736), como François Gigot de la Peyronie (1678-1747), los dos primeros cirujanos del Rey, lucharon contra la forma en que se practicaba el arte de curar. Consiguieron que únicamente pudieran practicar la Cirugía los que hubiesen adquirido el título de «maître des arts». Entre los directores de la Real Academia, hay que citar a Jean-Louis Petit (1674-1750). Fue inventor de un manguito para comprimir las arterias, describió las anomalías de la mano y del carpo, las luxaciones medio-carpianas, así como un método de amputación digital con dos colgajos. En cuanto a De La Peyronie, describió la induración fibrosa de los cuerpos cavernosos del pene, a la que llamó «strabisme pénin», a causa de las desviaciones que provoca, y que hoy se asocia en algunos casos con la enfermedad de Dupuytren.

Recorriendo los cinco primeros volúmenes de las Memorias de la Academia, encontramos varias observaciones de dedos arrancados, descritos por Recolin en 1735 y por Morand en 1736. Este último, recuerda una observación más antigua de Pierre de Marquetis, en 1665, de un pulgar arrancado por mordisco de caballo, con su tendón flexor pollicis longus entero. M. Planque, cirujano mayor del hospital Militar de Lille, envió en 1744 la observación de un soldado francés de la última guerra con Alemania., con un arrancamiento del pulgar entre primera y segunda falange, al que quedó adherido la totalidad del tendón extensor pollicis longus. Un caso parecido es el descrito por Malaese en 1749: el arrancamiento del anular derecho de una mujer, con su flexor pollicis longus entero.

Petrus Camper (1722-1789), aplica las nociones de Anatomía a las artes plásticas. Así concibió su famoso «ángulo facial». Pero por lo que aquí nos interesa es porque observa el quiasma de cruce del flexor digitorum superficialis por detrás del flexor digitorum profundus.

Astley Paston Cooper (1768-1814) es, quizás, uno de los principales representantes de la cirugía británica de la primera mitad del siglo XIX. La disección ocupó gran parte de su actividad. Describió la retracción de la aponeurosis palmar diez años antes de que lo hiciera Dupuytren.

En la época de las guerras napoleónicas, son descritas numerosas amputaciones, recomendadas por Larrey (1766-1842), con el propósito de evitar la gangrena gaseosa. Por ello se produce un enorme avance en la producción de prótesis y otros aparatos ortopédicos. 
En su «Traité d'opérations nouvelles, et inventions de mécanique, servant de moyens secondaires pour en assurer le succès», $\mathbf{P}$. Dutertre, relata diversas operaciones practicadas en manos accidentadas, así como el tratamiento de las secuelas de quemaduras, cicatrices retráctiles, que trataba por incisión de las bridas, inmovilizándo los dedos en extensión hasta su cicatrización, adelantándose así, en cierto modo, al método operatorio de Mc Cash, en el tratamiento quirúrgico de la enfermedad de Dupuytren, método bien conocido de todos los cirujanos de la mano actuales.

En este siglo XIX empiezan a surgir nombres de cirujanos famosos en varios países y los descubrimientos se suceden a un ritmo extraordinario. Así en Italia Giovanni Battista Monteggia (1762-1815), catedrático y cirujano de Milán, describe la fractura que lleva su nombre. En Alemania, Ferdinand von Graefe (17871840) fabrica prótesis de mano con dedos móviles, animados por tirantes de tripa. El austriaco Zeller (1746-1816) describe en 1810 la operación para la sindactilia. El irlandés Abraham Colles (1773-1843), es conocido por la fractura de muñeca que lleva su nombre, descrita en 1814. El barón Guillaume Dupuytren (17771835) nos deja la descripción de la fibrosis de la aponeurosis palmar que, aunque es conocida por su nombre, fue ya descrita por Cooper 10 años antes, como ya hemos visto. Joseph Swan (1791-1874), cuyos trabajos fueron citados por Boyes en 1976 en su libro «Notable names in Hand Surgery», fue cirujano en el Lincoln Hospital y fue, sin duda, el primero en experimentar en animales sobre heridas nerviosas periféricas. Ernst Heinrich Weber (1795-1878), en Alemania, describe su famoso test de la sensibilidad por dos puntos en 1834. Otro inglés, Charles Bell (1774-1842) formula los principios anatómicos en relación con la función, mientras que en Francia, Guillaume Duchenne De Boulogne (1806-1875), publica en 1867 su libro «Physiologie des mouvements», basado en la experimentación eléctrica, para explicar el estudio de las parálisis y los movimientos. Se le considera pionero en la Neurología y en la fotografía médica. No se pueden olvidar los nombres de Filippo Pacini (1812-1883), anatomista italiano. Descubrió los corpúsculos que llevan du nombre en 1831, cuando aún era estudiante en la Escuela
Médica de Pistoia, pero no los presentó hasta 1835. Otro nombre famoso es el de Friedrich Theodor Schwann (1810-1882), sobre todo por la descripción de las células envolventes del nervio, conocidas con su nombre. En Alemania hay que recordar a Augustus Volney Waller (18161870), por sus trabajos sobre la degeneración del axón seccionado y su regeneración central («la regeneración valeriana»). Alphonse-Henri Notta (1824-1914) describe en 1850, en el dedo medio de la mano derecha, lo que más tarde su maestro Auguste Nelaton (1807-1873) llamará el «dedo en resorte» en 1859. James Paget (1814-1899), se ocupa sobre todo de las enfermedades óseas y describe la que lleva su nombre, William Adams (1820-1900), conocido por la «línea de Adams», entre el trocánter menor y el borde inferior del cuello del fémur, trabajó sobre las parálisis de los miembros. Y una serie de nombres bien conocidos, como Raynaud (1834-1881), Volkmann (1830-1889), Weir Mitchell (1829-1914). Y cómo olvidarnos de Esmarch (1823-1908), sin cuya banda hemostática nos sería imposible practicar la cirugía de la mano. Harold Bennett (1837-1907), bien conocido por la fractura-luxación de la base del primer metacarpiano, descrita en 1882, antes del descubrimiento de los rayos $\mathrm{X}$.

En Suiza, el sucesor en Berna de Théodor Kocher (1841-1917), que obtuvo el Premio Nobel de Medicina en 1909, Fréderich de Quervain (1868-1940) describe en 1895, a los 27 años, la tenosinovitis estenosante del abductor pollicis longus y extensor pollicis brevis, conocida con su nombre. Aunque, al parecer ya había sido descrita en 1880 por Tillaux, como el «ay» crepitante, y en el tratado de anatomía de Gray en 1893, como «esguince de la lavandera».

En Viena, Carl Nicoladoni (1847-1902), reconstruye el pulgar amputado de un muchacho, con los elementos procedentes de un segundo dedo del pie. Ya en 1868, Charles Huguier (1804-1873), buscaba reconstruir un pulgar con elementos residuales de la mano gravemente herida. Basándose en sus ideas, François Guermonprez (1849-1932) publica en 1887 un trabajo sobre las «resecciones y restauraciones del pulgar». Es el precursor de la implantación de un dedo largo de la misma mano sobre la base de un pulgar amputado. 
Fue en 1895 cuando el físico alemán Konrad Röntgen (1845-1923) descubre los rayos X. Hizo la presentación de su descubrimiento en Wurzburg, en la Academia de Ciencias, presentando una radiografía de la mano de su esposa.

Entrando ya en el siglo XX, precisamente en 1901, Henri-François Secretan (1856-1916), describe el edema duro e hiperplasia traumática del dorso de la mano, en ocasiones sospechoso de auto-mutilación, pero en muchos casos de origen traumático no voluntario. En 1903 Paul Sudeck (1866-1945), describe su «Akute Knochenatrophie» que, según propuso más tarde Marc Iselin, se llamó «algodistrofia refleja» y hoy en día «síndrome doloroso regional complejo».

En los EE.UU., Allen Kanavel (1874-1938), estudia los espacios celulosos profundos de la mano y propone en 1912, en su libro «Infections of the Hand», nuevas incisiones.

Robert Kienböck (1871-1953), radiólogo de los Hospitales de Viena, describe en 1910, la necrosis aséptica del hueso semilunar, llamada desde entonces «Enfermedad de Kienböck».

Dos años más tarde, en 1912, William Darrach (1871-1948) demuestra la posibilidad de hacer una resección sub-periostica de la cabeza del cúbito en las dislocaciones por fractura de la muñeca

También, en 1912, el gran cirujano suizo Cesar Roux (1857-1934) hizo una tentativa de pulgarización de una anular, implantándolo al revés en el muñón restante del pulgar. Leo Mayer (1884-1972), futuro fundador del Hospital for Joint Disease de Nueva York, publica en alemán, durante su estancia en Munich, una monografía sobre la cirugía de los tendones, en la que la formación y la prevención de las adherencias está ya entre sus principales preocupaciones. Pierre Marie (1853-1893) y Charles Foix (1882-1927) publican en 1913 la primera descripción del síndrome de compresión del nervio mediano en el túnel carpiano.

Martin Kirschner (1879-1942) inventa las famosas agujas para fijación de fracturas de la mano, que tanto se siguen utilizando en la actualidad. Del mismo modo, el suizo Fritz Steinmann, en 1908 publica un artículo sobre su famoso clavo.

Estamos en vísperas de la Primera Guerra Mundial, en la que empezará a tomar una enor- me importancia el tratamiento de las lesiones traumáticas de la mano, como consecuencia de las terribles secuelas que las lesiones por armas de fuego pueden provocar. Así, las lesiones de los nervios periféricos son numerosas, lo que lleva a que, por ejemplo, Jules Froment (1878-1946), Profesor de Neurología en Lyon, sea encargado de una unidad especializada en Rennes. Analiza y precisa los signos de las diversas lesiones nerviosas y describe en 1915 su «signo del periódico» para el diagnóstico de la parálisis cubital. Un año más tarde, Jules Tinel (1879-1952) da a la luz su libro titulado «Las heridas de los nervios. Semiología de las lesiones nerviosas periféricas por herida de guerra», donde describe la regeneración del nervio seccionado y suturado. De todos es conocido su famoso «signo del hormigueo» a la percusión de un neuroma. Otro gran cirujano militar durante la Primera Guerra Mundial es, sin duda, Lorentz Böhler (1885-1973), que muchos historiadores consideran como el creador de la moderna Traumatología. Publicó su «Tratado de las Fracturas» en 1929. También en el campo germánico hay que resaltar la figura de Ferdinand Sauerbruch (1875-1951), Profesor de Clínica Quirúrgica en Zurich, quien tuvo la idea de animar una prótesis de mano y antebrazo, sirviéndose de los músculos del brazo. Hay que destacar también al alemán Hermann Krukenberg (1863-1935), que ideó en 1917 su famosa falangización de los huesos del antebrazo en amputados por debajo del codo, permitiendo una pinza sensible y activa, gracias a la función del pronator teres.

Se adquirió muchísima experiencia que figuró en numerosas obras, como la de Sir Harold Stiles (1863-1936), de Edimburgo, en la que describe por vez primera la anastomosis del tendón digitorum superficialis sobre el aparato extensor de los dedos con el fin de corregir la «garra cubital». En esa época se crea en Inglaterra la Cirugía Ortopédica como especialidad con Sir Robert Jones (1855-1933), autor de la utilización del pronator teres, como motor de los radiales en la parálisis del nervio radial. Se decía que cuando Jones operaba el tiempo se detenía, tal era su habilidad. En Italia, Ricardo Galeazzi (1866-1952), de Milán, describe la fractura de antebrazo que lleva su nombre. 


\section{NACIMIENTO DE UNA NUEVA ESPECIALIDAD}

Como «no hay mal que por bien no venga», es seguramente durante uno de los mayores males de la Historia: la Segunda Guerra Mundial, que la Cirugía de la Mano alcanza un desarrollo extraordinario, sobre todo gracias a Sterling Bunnell (1882-1957), cirujano general, aunque ya publica su primer trabajo sobre tendones flexores en 1918. Creó una escuela en San Francisco, de la que nacen numerosos especialistas en EE.UU. y en el mundo entero. Otra escuela es creada en Chicago por Kanavel, del que ya hemos hablado, seguido por Sutnner Koch, Michel Mason y Harvey Allen. También en Paris, la cirugía de la mano empezó a considerársela de capital importancia desde 1928, gracias a Marc Iselin (1898-1987), cirujano general, sobre todo de la región torácica, pero muy conocido por su especial interés en la Cirugía de la Mano, que la hace conocer en toda Europa gracias a dos libros, uno destinado al cirujano, otro al gran público. Son sus directivas las que Claude Verdan (1909-2006), se esfuerza en aplicar durante la guerra en el Servicio de Cirugía del Hospital Cantonal de Lausana.

Dice Verdan que durante el periodo en que fue Médico-Jefe responsable de la Caja Nacional Suiza de Seguros en caso de Accidentes (de 1938 a 1944), pudo peritar un gran número de manos mutiladas, pudiendo constatar cómo esta cirugía era poco conocida, mal aplicada e incluso despreciada. Así, cuando en 1944 su Maestro, el Profesor Pierre Decker, le confió la consulta ambulatoria del Servicio Universitario de Cirugía, se dio cuenta de que todo estaba por hacer en esta materia. Fue allí donde empezó a poner en práctica lo que sería, desde entonces, su preocupación mayor: la Cirugía de la Mano. En sus «Memorias de un Cirujano», editadas por la Fundación Claude Verdan de Lausanne, describe el estado lamentable en que se encontraba esta cirugía en Suiza: todo dedo rígido era amputado, los panadizos eran tratados con antiflogísticos y baños calientes. Después de noches en blanco, se utilizaba el método inspirado de Kanavel: incisiones medio-laterales escalonadas a lo largo del dedo, pasadas en túnel de parte a parte y mantenidas abiertas por medio de láminas de caucho. No eran infrecuentes las lesiones de los pedículos vásculo-nerviosos. En aquella época, los antibióticos eran aún desconocidos en Suiza, únicamente las primeras sulfamidas acababan de nacer. Y así, con esa negligencia e ignorancia, se trataba todo en la mano. Por ejemplo, las lesiones tendinosas se trataban de forma grosera, con resultados catastróficos; a veces con injertos del flexor digitorum profundus para el superficialis. Verdan no recuerda haber presenciado un solo buen resultado. Es por todas estas razones por las que Verdan se puso como objetivo crear su propia clínica, lo que ocurrió poco tiempo después, en noviembre de 1946, bajo el nombre de «Permanence médico-chirurgicale et Clinique de Longeraie», lugar en el que, algunos años más tarde, el autor de este artículo ha pasado la casi totalidad de su vida profesional como cirujano de la mano.

Durante esos años, en los Estados Unidos, Bunnell había explorado la cirugía de la mano de forma excepcional y está listo, en 1944, cuando el médico jefe de los ejércitos americanos le encarga de los cuidados de numerosas manos heridas durante la guerra. Ese mismo año ve la luz su libro «Surgery of the Hand», desconocido por los europeos, pero considerado por los cirujanos norteamericanos «como una nueva luna en el cielo», según Joseph H. Boyes (1905-1995), uno de sus seguidores.

Es a partir de estos años 40, cuando la Cirugía de la Mano se fue extendiendo por todo el mundo. La publicación en 1948 de una segunda edición del libro de Bunnell, traducido ya a otras lenguas, fue una verdadera revelación en toda Europa. Hay que señalar también la importancia que tuvieron desde 1954, los cursos de perfeccionamiento en cirugía de la mano de Marc Iselin en Nanterre y los dados por Robert Merle D’Aubigné (1900-1989) y su escuela en el Hospital Cochin de Paris, en especial Raoul Tubiana, de todos conocido.

Empiezan a publicarse muchos libros consagrados a la cirugía de la mano, entre otros el del español Mario Oliveras en 1948 «Traumatismos e Incapacidades de la Mano»; o el de Eugenio T. López-Trigo, Jefe del Servicio de Traumatología del Hospital Provincial de Valencia: «Lesiones Osteo-Articulares Traumáticas de la Muñeca», también publicado en 1948. El de 
E. Velilla Mateo, del Hospital de la Princesa de Madrid: «Traumatologçía y Cirugía Reparadora de la Mano», de 1950. En Suiza, Verdan, en 1952, publica: «Chirurgie Reparatrice et Fonctionnelle des Tendons de la Main»

Entre 1945 y 1968 se publicaron, según Verdan, veintiocho obras consagradas a la Cirugía de la Mano.

Empiezan a constituirse pequeños grupos de estudio dedicados a esta materia, que se transformarán más tarde en verdaderas sociedades nacionales. Así, en 1946, inmediatamente después de la Segunda Guerra Mundial, 35 cirujanos norteamericanos bajo el impulso de Bunnell, se congregan en Chicago, convocados por Boyes, en asamblea constituyente de la American Society for Surgery of the Hand (ASSH), bajo la presidencia de Sterling Bunnell. Eric Moberg (1905-1993), en 1953 fundó la «Scandinavian Society for Surgery of the Hand» y consiguió, en 1969, que la cirugía de la mano fuera reconocida en Suecia como especialidad quirúrgica a parte entera por las autoridades suecas, razón por la que, unidades de Cirugía de la Mano, fueran creadas en todas las Universidades del país. Aún hoy en día, según mis informaciones, solamente Suecia, Finlandia y Singapur gozan de este privilegio.

En Inglaterra, se crea en $1952 \mathrm{el}$ «Hand Club». Se trataba de un grupo de 12 miembros que se reunían un viernes por la noche a cenar, celebrando una sesión clínica el sábado, en el Hospital que, por rotación, correspondía a cada uno de los miembros. Entre este grupo se encontraba Guy Pulvertaft (1907-1986). Era un grupo muy cerrado donde, por ejemplo, rehusaron la entrada a Graham Stack. Con otros en la misma situación, se creó el «Second Hand Club», en una reunión en 1956. Estaban presentes, entre otros, Douglas Campbell y Adrian Flatt. Bajo la impulsión de Pulvertaft, se reunieron los dos «Clubs»y prepararon la creación de la «British Society for Surgery of the Hand», lo que se confirmó en mayo de 1969, teniendo como primer presidente a Pulvertaft. Entre sus posteriores presidentes, hubo uno no inglés (Raoul Tubiana en 1974) y uno no cirujano, sino fisioterapeuta de renombre mundial (Kit Wynn-Parry en 1982).

Transcurría el año 1959, cuando Dieter Buck-Gramcko, brillante cirujano de Hambur- go, se puso en contacto con Verdan, con el fin de que participase en la formación de un grupo de estudio de la mano de lengua alemana, con el fin de crear una sociedad común a varios países de la misma lengua. La primera reunión de este grupo, a la que no asistieron más de una decena, tuvo lugar en 1960. Así nació la «Deutsche Arbeitsgemeinschaft für Handchirurgie» (DAH), cuyas reuniones anuales fueron siempre de gran interés.

También en Italia, en 1962, se creó una gran sociedad, la «Società Italiana di Chrurgia della Mano), bajo el impulso de Augusto Bonola, de Modena y cuyos socios fundadores fueron, además de Bonola, Giorgio Brunelli, Aldo de Negri, Leonardo Gui, Germano Mancini, Humberto Mangini, Enzo Morelli y Filippo Perricone. Publica su primer número de la «Rivista Iitaliana di Chirurgía della Mano» el 22 de enero de 1964 . He de agradecer también a esta Sociedad el que rápidamente me acogiese como miembro de la misma. Hoy en día es probablemente una de las más numerosas en número de miembros y una de las más activas.

En Francia, alrededor de Marc Iselin, se forma la «Association pour la Sauvegarde de la Main» y alrededor de Merle d'Aubigné, con su alumno Raoul Tubiana y Jacques Duparc, Jacque Michon, Raymond Vilain y Pierre Rabichon se constituye en 1964 un grupo de estudio de la mano (GEM), núcleo de origen de la «Société Française de Chirurgie de la Main», de la cual tengo también el honor de ser miembro.

No quiero olvidar a un grupo de amigos, la mayor parte alumnos de Marc Iselin en Nanterre, como Levame, Recht, Haimovici, Mantero, Allieu, Gosse, etc. que constituyeron lo que se decidió en llamar «Amicale Internationale de Chirurgie de la Main», organizando reuniones donde no se preparaban conferencias, donde se discutían casos interesantes, donde no había protagonismos, pero en la que se llegaba al fondo de los problemas. Asistí a varias reuniones, pero desgraciadamente no duró mucho.

La primera reunión nacional sobre Cirugía de la Mano en España, la organiza en Zaragoza Alfredo Quintana Montero en febrero de 1969. Cuenta con la participación, como invitados, de Pulvertaft, el finlandés Kauko Vainio, Tubiana y Souquet, de Toulouse. Y entre los españoles, 
Vicente Senti Montagut, Jaime Planas y Fernando Enríquez de Salamanca. Como consecuencia de aquella reunión, se decidió crear la Sociedad Española de Cirugía de la Mano (SECMA), con 29 miembros. Yo tuve la ocasión de participar, como invitado, en la tercera reunión nacional, que se celebró en Zaragoza en junio de 1974, junto a Yves Allieu, de Montpellier, A. Chancholle, Nicolas Haimovici, Ian Matev, Aldo de Negri, A. Pannique, Enzo Morelli y Kit Wynn-Parry. También tengo que agradecer a esta Sociedad, que es la mía, el que me concediera el gran privilegio de nombrarme Miembro de Honor en diciembre de 1993.

En Suiza, bajo el influjo de varios cirujanos generales que tenían que afrontar con frecuencia heridas en las manos, se decidió Verdan, el 12 de noviembre de 1966, a celebrar una asamblea constituyente, con una cincuentena de participantes, para la creación de un «Groupe Suisse d'Études de Chirurgie de la Main» (GSECM). No se formó ningún comité de dirección, sino que se nombró un secretario general en la persona de Verdan, puesto que ocupó durante seis años, pasando después a ocuparlo Henry Nigst, de Basilea. Años más tarde, bajo el impulso de Algimantas Narakas, este grupo pasó a constituir una sociedad más estructurada: la «Société Suisse de Chirurgie de la Main».

También en Sudamérica se manifestó, bajo la influencia de los norteamericanos, un gran interés por la Cirugía de la Mano. Así, el 2 de diciembre de 1965, en la ciudad de Lima (República de Perú), se funda la Sociedad Sudamericana de Cirugía de la Mano (Habla hispana), «con la finalidad de divulgar, jerarquizar y perfeccionar vínculos y relaciones científicas con entidades similares y con los cultores de dicha especialidad en Sudamérica y en todo el mundo». Los fundadores fueron: Teodoro Ayala (Paraguay), Enrique Yenkin (Chile), Edgard Kamel (Venezuela), Eugenio Elizalde (Perú), Carlos Firpo (Argentina) y Eduardo A. Zancolli (Argentina), siendo éste último nombrado Presidente.

En 1966, ocho Sociedades nacionales decidieron formar una Federación Internacional, lo que se logró, con la llegada de nuevos miembros, en 1969, creando así la Federación Internacional de Sociedades de Cirugía de la Mano (IFSSH). Más tarde, en 1991, vio la luz la Federación
Europea de Sociedades de Cirugía de la Mano (FESSH), sin duda para independizarse algo del poder de los norteamericanos.

Es probablemente la microcirugía, tanto de los nervios periféricos como la vascular, la que ha revolucionado de forma impresionante en los últimos años la Cirugía de la Mano. Permite realizar colgajos libres de diversos complejos tisulares, tomados del mismo individuo, como la utilización de un dedo de pie para reconstruir un pulgar amputado. Solamente la utilización del microscopio ha permitido el reimplante de dedos, e incluso manos, amputados.

Sin embargo, debo aquí emitir mis reservas a algunas prácticas actuales, motivadas más - a mi modo de ver - por una cierta publicidad en los medios de comunicación, que por el bien del paciente. Me quiero referir, por ejemplo, a la utilización de miembros de cadáveres en la reconstrucción de miembros amputados a nivel de antebrazo. En un amputado bilateral podría, en último caso ser de alguna utilidad, pero siempre con el absoluto acuerdo del paciente, una vez informado de los peligros a que se le va a someter, del prolongadísimo tratamiento, tanto medicamentoso como de rehabilitación, de la certeza de que nunca recobrará una función ni una sensibilidad normales. En un amputado unilateral, creo que este procedimiento no debería ser aplicado. Con un miembro superior normal y una prótesis mio-eléctrica en el miembro amputado, el resultado funcional será superior.

No olvidemos el importante avance que significó la utilización de nuevas sustancias plásticas en silicona. Permitieron a investigadores, como Alfred Swanson de Grands Rapids (EE.UU.), desde 1962, la creación de implantes flexibles, destinados a la remodelación de articulaciones de la mano o de la muñeca, destruidas o funcionalmente alteradas por traumatismo o por enfermedad reumatoide. Varillas flexibles de la misma materia, permitieron a J. Hunter, de Filadelfia (EE.UU.), reconstruir vainas tendinosas destinadas a recibir, en dos tiempos, injertos de flexores en dedos destinados, en otras épocas, a la amputación.

Finalmente, las técnicas quirúrgicas con métodos endoscópicos, utilizadas por cirujanos bien preparados y experimentados, pueden aportar un cierto beneficio, al disminuir el periodo de cicatrización y de recuperación funcional. Pero, 
también aquí, tengo la impresión de que su banalización comporta muchos más riesgos que las intervenciones a cielo abierto.

No quiero, ni puedo, prolongar más adelante este acercamiento a la Historia de la Cirugía de la Mano, porque temo dejar de lado hechos y personajes importantes en esta materia. Espero que sirva de guía a cuantos jóvenes cirujanos se inclinen por esta maravillosa especialidad, que ha significado para este miembro de esa gran familia, lo esencial de su vida profesional.
Para finalizar, quiero recordar aquí los principios que, según mi modo de entender la Cirugía de la Mano, deben guiarnos en el momento de encontrarnos cara a cara con una mano mutilada, que necesita de nuestra ayuda:

\author{
LÓGICA EN LA DECISIÓN \\ DELICADEZA EN LA EJECUCIÓN \\ HUMANIDAD EN EL CONTACTO \\ $\mathrm{Y}$ \\ MODESTIA... SIEMPRE
}

